\title{
Role of the open-shell character in the pressure-induced conductivity in an organic donor-acceptor radical dyad
}

\author{
Manuel Souto, ${ }^{[\mathrm{a}]}$ Maria Chiara Gullo, ${ }^{[\mathrm{a}]}$ HengBo Cui,${ }^{[\mathrm{b}]}$ Nicola Casati, ${ }^{[\mathrm{c}]}$ Fabio Montisci, ${ }^{[\mathrm{d}]}$ Harald O. \\ Jeschke,${ }^{[\mathrm{e}]}$ Roser Valentí, ${ }^{[\mathrm{e}]}$ Imma Ratera* ${ }^{[\mathrm{a}]}$ Concepció Rovira, ${ }^{[\mathrm{a}]}$ and Jaume Veciana ${ }^{*[\mathrm{a}]}$
}

\begin{abstract}
Single-component conductors based on neutral organic radicals have received a lot of attention due to the possibility that the unpaired electron can serve as a charge carrier without the need of a previous doping process. Although most of these systems are based on delocalised planar radicals, we present here a nonplanar and spin localised radical based on a tetrathiafulvalene (TTF) moiety, linked to a perchlorotriphenylmethyl (PTM) radical by a conjugated brige, which exhibits a semiconducting behavior upon application of high pressure. The synthesis, electronic properties and crystal structure of this neutral radical TTF-Ph-PTM derivative (1) are reported and implications of its crystalline structure on its electrical properties dicussed. On the other hand, the non-radical derivative $\mathbf{2}$, which is isostructural with the radical $\mathbf{1}$, shows an insulating behavior at all measured pressures. The different electronic structures of these two isostructural systems have a direct influence on the conducting properties as demonstrated by band structure DFT calculations.
\end{abstract}

Organic molecular materials exhibiting electrical conductivity, normally based on two components such as charge transfer salts, is a research field that has received a lot of attention during the last decades. ${ }^{[1]}$ More interesting is the development of single-component conductors since they offer the possibility for three-dimensional electronic structure because the absence of any counterion. ${ }^{[2]}$ However, realisation of purely-organic singlecomponent conductors is a major challenge since organic materials are normally insulating due to the full-filled valence band and the large energy band between the valence and conduction bands. From a molecular point of view, these two reasons are equivalent to the closed-shell electron configuration of the HOMO and the large excitation energy from the HOMO to the LUMO. Thus, in order to produce single-component purelyorganic molecular conductors it is necessary to remove electrons from the HOMO (or add electrons to the LUMO) and reduce the HOMO-LUMO energy gap.

\footnotetext{
[a] Dr. M. Souto, M.C. Gullo, Dr. I. Ratera, Dr. C. Rovira, Dr. J. Veciana Institut de Ciència de Materials de Barcelona (ICMABCSIC)/CIBER-BBN

Campus Universitari de Bellaterra, 08193, Bellaterra, Spain E-mail: vecianaj@icmab.es

[b] Dr. H. Cui

Condensed Molecular Materials Laboratory, RIKEN,

Wako-shi, Saitama, 351-0198, Japan

[c] Dr. N. Casati

Paul Scherrer Institut

WLGA/229, CH-5232 Villigen PSI, Switzerland

[d] F. Montisci

Department of Chemistry and Biochemistry, University of Bern Freiestrasse 3, 3012 Bern, Switzerland

[e] Dr. H. O. Jeschke, Dr. R. Valentí

Institut für Theoretische Physik, Goethe-Universität Frankfurt

Max-von-Laue-Straße 1, 60438 Frankfurt am Main, Germany
}

The first single-component metal was reported by Kobayashi et al. in 2001 based on a Nickel complex in which conductive $\pi$ electrons are delocalised over the tetrathiafulvalene(TTF)-type large ligand skeleton decreasing the HOMO-LUMO gap. ${ }^{[3]}$ There are also many $\mathrm{Au}$ bis(dithiolene) complexes with singlecomponent conductor behaviour ${ }^{[4-6]}$ and in some of them additional properties of chirality have been introduced. ${ }^{[7]}$ Mori et al. have recently reported some purely-organic singlecomponent conductors based on TTF-based molecules functionalised with a catechol unit which exhibit high electrical conductivity due to the hydrogen bond-promoted delocalisation of charge carriers upon partial oxidation of the molecules. ${ }^{[8,9]}$ More recently, a single-component and pure organic system based on a zwitterionic TTF-extended dicarboxylate radical exhibiting very high metallic conduction in a broad range of temperatures has been reported. ${ }^{[10]}$

On the other hand, neutral organic radicals have also been proposed as pure organic single-component conductors since the unpaired electron can serve as a charge carrier without the need of a previous doping process. ${ }^{[1]}$ Most of the reported examples are based on highly delocalised and planar systems, such as phenalenyl- and thiazolyl-based radicals, ${ }^{[12-15]}$ and some of them enhance the electrical conductivity with the compression of the crystal lattice under high pressure..$^{[16-19]}$

Recently, we have reported a new type of neutral nonplanar and spin-localized radical conductor which is based on a monopyrrolo(MP)-TTF unit linked to a perchlorotriphenylmethyl (PTM) radical through a phenyl-vinylene bridge (MPTTFPTM). ${ }^{[20]}$ This system is insulating at ambient pressure and becomes semiconducting at high pressure due to the increased electronic bandwidth $W$ and charge reorganization which decreases the Coulomb energy repulsion $U$. This charge reorganization is occurring in the solid state through an intramolecular charge transfer (ICT) between the electron-donor TTF unit and the electron-acceptor PTM radical through the conjugated bridge; as it has been observed in solution for many other similar systems. ${ }^{[21-24]}$ However, until now it has not been possible to study in detail the effect of the electronic structure since the open- and closed-shell derivatives crystallized in a different manner. ${ }^{[25]}$

In order to study the effect of both the open-shell structure and the bridge length, we have designed and synthesized a shorter dyad in which both donor and acceptor units are linked through a phenyl-vinylene bridge (TTF-Ph-PTM) (1, Scheme 1). In addition, we have also prepared the non-radical analogue (TTF-Ph-PTM-H; 2), which shows the same crystal structure as 1, in order to study, for the first time, the influence of the electronic structure (open- versus closed-shell) on the conducting properties. Interestingly, radical dyad 1 becomes semiconductor from $11 \mathrm{GPa}$ whereas the isostructural nonradical dyad 2 remains insulating at all measured pressures demonstrating unambiguously the key role played by the radical character of dyad 1. Band structure calculations based on 
density functional theory (DFT) confirm that $\mathbf{1}$ shows a significant smaller bandgap than 2 which explains the different conducting behavior.

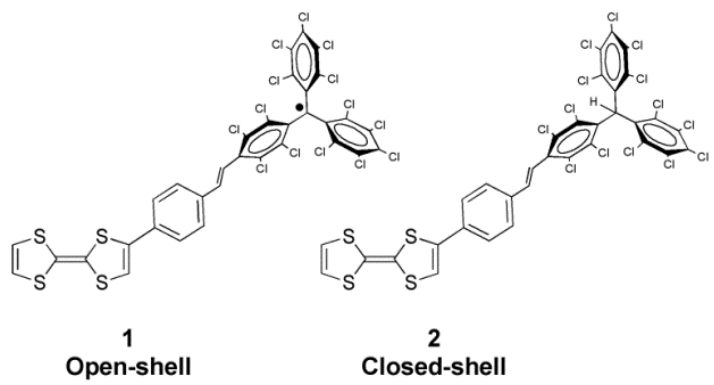

Scheme 1. Molecular structure of radical dyad 1 and non-radical dyad 2.

Initially, we describe the synthesis of the TTF aldehyde precursor which is based on a palladium-catalyzed direct $\mathrm{C}-\mathrm{H}$ arylation of TTF with the corresponding aryl bromide. ${ }^{[26]}$ Treatment of TTF (3) with 4-bromobenzaldehyde (4) in presence of cesium carbonate and catalytic amounts of palladium acetate and tri-tert-butylphosphonium tetrafluorobrate in refluxing THF provided the TTF-Ph-CHO (5) (Scheme 2) in 22\% yield after purification by column chromatography.

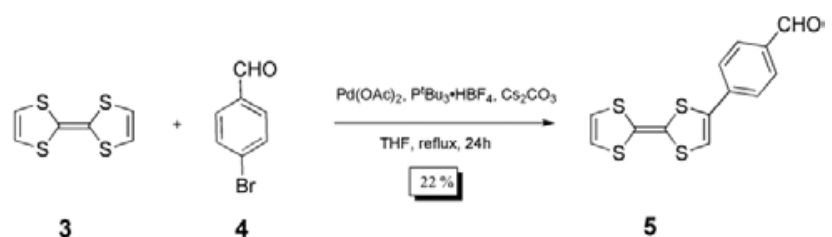

Scheme 2. Synthesis of TTF-Ph-CHO precursor 5.

The syntheses of dyads $\mathbf{1}$ and $\mathbf{2}$ is summarised in Scheme 3. First, non-radical dyad 2 was obtained as a red solid through a Horner-Wadsworth-Emmons reaction of compound $\mathbf{5}$ and the phosphonated PTM derivative $6^{[27]}$ in $30 \%$ yield. Then, the subsequent deprotonation of 2 with $\mathrm{TBAOH}$ and oxidation of formed carbanion with $\mathrm{AgNO}_{3}$ yields radical dyad $1(66 \%)$ as a black solid and stable under ambient conditions (Scheme 3).

Electrochemical properties of dyads $\mathbf{1}$ and $\mathbf{2}$ were studied by cyclic voltammetry $(\mathrm{CV})$ that was performed in $\mathrm{CH}_{2} \mathrm{Cl}_{2}$ at room temperature (Figure 1 ). Non-radical dyad 2 exhibits two redox waves that are assigned to the step-wise oxidation of the TTF moiety to TTF ${ }^{++}$and TTF $^{2+}$ with redox potentials at 0.39 and $0.92 \mathrm{~V}$, respectively. On the other hand, radical dyad 1 exhibits three redox waves assigned to the redox processes PTM-/PTM-, $\mathrm{TTF}_{\mathrm{TTTF}}{ }^{+}$and $\mathrm{TTF}^{+} / \mathrm{TTF}^{2+}$ at $-0.16,0.44$ and $0.97 \mathrm{~V}$, respectively. The oxidation potentials related to the oxidation of the TTF moiety shift $0.05 \mathrm{~V}$ towards more positive potentials from 2 to 1 indicating a stronger interaction between the donor and acceptor groups in $\mathbf{1}^{[23,24]}$ The estimated disproportionation $\left(E_{\text {disp }}\right)$ potential in solution can be calculated from the difference between the first oxidation and reduction potentials and provide an indirect measurement of the Coulomb repulsion energy $U$, that gauge the energy needed to transfer intramolecularly one electron from the donor to the acceptor site. The estimated disproportionation potential of radical dyad $\mathbf{1}$ is $0.60 \mathrm{~V}$ which is only slightly lower than that of the related MPTTF-PTM dyad $(0.64 \mathrm{~V})^{[20]}$ suggesting a similar Coulomb energy repulsion for both systems.

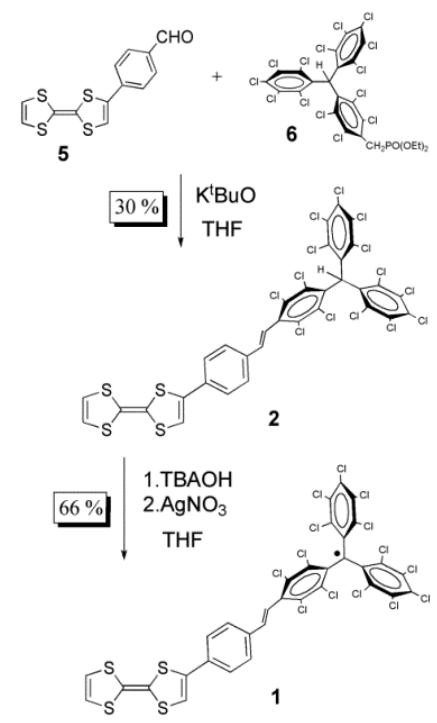

Scheme 3. Synthesis of dyads 5 and 2.

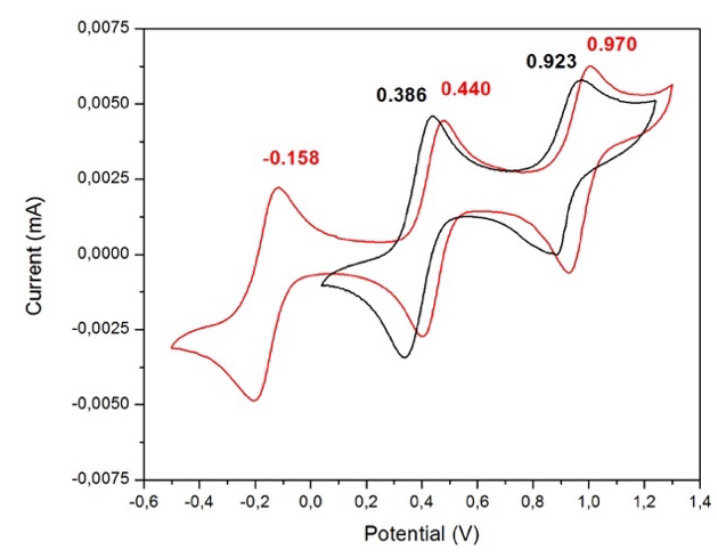

Figure 1. Cyclic voltammogram of radical dyad $\mathbf{1}$ (red line) and $\mathbf{2}$ (black line) in $\mathrm{CH}_{2} \mathrm{Cl}_{2}$ versus $\mathrm{Ag} / \mathrm{AgCl}$ using $\mathrm{TBAPF}_{6}$ as electrolyte, under Argon and scan rate of $0.1 \mathrm{~V} / \mathrm{s}$.

The UV-vis-NIR spectra of dyads $\mathbf{1}$ and $\mathbf{2}$ were recorded in $\mathrm{CH}_{2} \mathrm{Cl}_{2}$ at $300 \mathrm{~K}$ (Figure 2). The spectrum of non-radical dyad 2 shows two principal bands at 318 and $430 \mathrm{~nm}$. On the other hand, absorption spectrum of radical dyad 1 shows an additional intense band at $385 \mathrm{~nm}$, characteristic of PTM radical chromophores, and two-less resolved peaks at 440 and $550 \mathrm{~nm}$ 
attributed to the delocalization of the unpaired electron into the conjugated framework. In addition, a broad absorbance is observed in the 700-1000 $\mathrm{nm}$ range which is assigned to an intramolecular charge transfer (ICT) process from the TTF to the PTM radical as it has been observed in other similar TTF-PTM systems. ${ }^{[21,23,24]}$ Thus, the optical gaps calculated from the lowest-energy bands for dyads 1 and $\mathbf{2}$ were found to be 1.2 and $2.0 \mathrm{eV}$, respectively, in agreement with the presence of the single unoccupied molecular orbital (SUMO) in radical dyad 1 which appears at lower energies than the LUMO of 2. $^{[24]}$

Absorption spectra of radical dyad $\mathbf{1}$ were measured in different solvents in order to compare the solvatochromic behavior observed in related TTF-PTM radical dyads. ${ }^{[21,23,28]}$ This solvatochromism is assigned to the bistability phenomenon between neutral and zwitterionic species since polar solvents can induce an intramolecular electron transfer process from the electron-donor TTF unit to the electron-acceptor PTM radical. In the case of dyad 1, this phenomenon of bistability was only observed in strong polar solvents such as DMF or DMSO with the appearance of a new band at $512 \mathrm{~nm}$ assigned to the anionic form of the PTM (Figure S2) and indicating the coexistence of neutral and zwitterionic species as it has been observed for the MPTTF-PTM dyad. ${ }^{[23]}$ Moreover, the absorption spectrum of 1 was compared with those of other related dyads (TTF-PTM ${ }^{[21]}$ and MPTTF-PTM ${ }^{[23]}$ ) observing that the ICT band was always in the same range of energies (Figure S3) and that the estimated HOMO-SUMO gap of $1(1.2 \mathrm{eV})$ is smaller than the one of MPTTF-PTM dyad (1.3 eV).

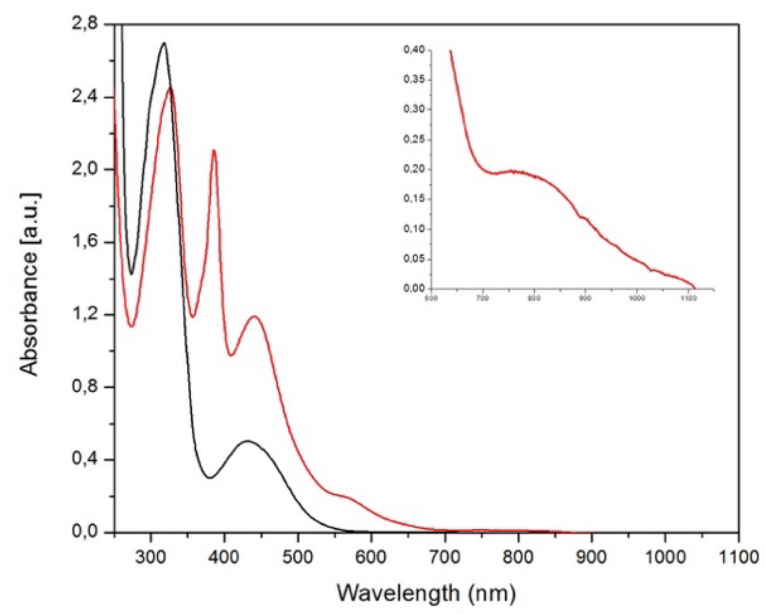

Figure 2. UV-vis-NIR spectrum of radical dyad 1 (red line) and $\mathbf{2}$ (black line) in $\mathrm{CH}_{2} \mathrm{Cl}_{2}(0.05 \mathrm{M})$ at $300 \mathrm{~K}$. The inset shows the low-energy range of the absorption spectrum of dyad 1.

Magnetic properties of radical dyad $\mathbf{1}$ were studied in solution by means of Electron Spin Resonance (ESR) (Figure 3). The ESR spectrum of dyad 1 was recorded in $\mathrm{CH}_{2} \mathrm{Cl}_{2}$ /toluene (1:1) at 220 $\mathrm{K}$ and shows at $g$-value of 2.004 two main lines with which corresponds to the hyperfine coupling of the unpaired electron with one ${ }^{1} \mathrm{H}$ of the vinylene bridge. The isotropic coupling constants showed the typical values for vinylene-substituted PTM radicals $\left(a_{\mathrm{H}}=1.9 \mathrm{~Hz}, \Delta H_{\mathrm{pp}}=1.1 \mathrm{~Hz}\right)$.
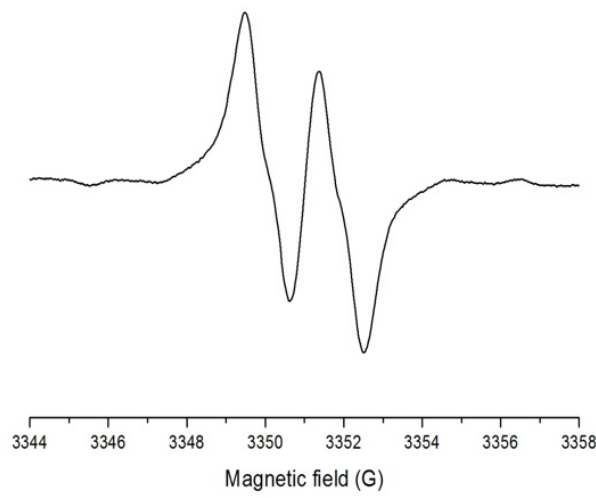

Figure 3. ESR spectrum of radical dyad 1 in $\mathrm{CH}_{2} \mathrm{Cl}_{2} /$ toluene (1:1) at $220 \mathrm{~K}$.

Dyads 1 and 2 were crystallized by a slow evaporation from a solution of hexane $/ \mathrm{CH}_{2} \mathrm{Cl}_{2}$. Both dyads are isostructural showing that they crystallized in the triclinic system with a $\mathrm{P}-1 \square$ space group and the unit cell parameters are $a=9.2 \AA, b=11.4 \AA$ and $c=19.1 \AA$. The fact that both dyads 1 and 2 have the same crystal structure is quite significant for the purpose of this study since the previously reported non-radical and radical MPTTFPTM dyads crystallized in a different manner. ${ }^{[20,23,25]}$ The molecular structure of $\mathbf{1}$ and $\mathbf{2}$ shows dynamic disorder in the phenyl ring and the TTF unit is distorted with respect to the phenyl and vinylene bridge which has a trans-configuration (Figure 4). The S2-C1-S1-C2 torsion angle in the TTF moiety is $7.7^{\circ}$ and the average of TTF $\mathrm{C}=\mathrm{C}$ bond length is $1.34 \AA$ indicating that the TTF moiety remains neutral in both cases. The main difference between the molecular structure of $\mathbf{1}$ and $\mathbf{2}$ is the hybridization of the central C of the PTM unit $\left(\mathrm{sp}^{2}\right.$ and $\mathrm{sp}^{3}$, respectively) with C18-C21, C28-C21 and C22-C21 distances of ca. 1.47 and $1.54 \AA$, for the radical and non-radical cases, respectively.

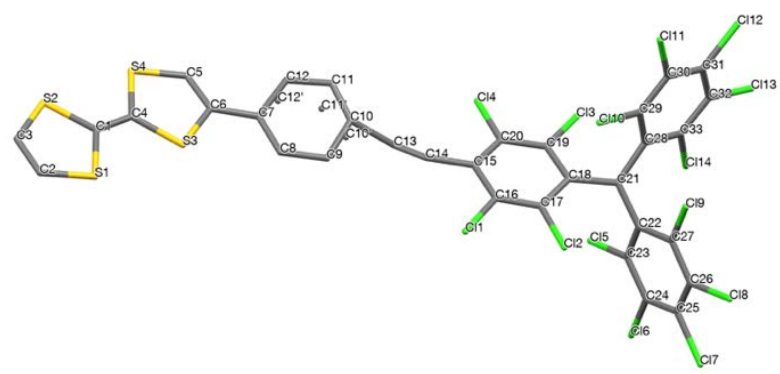

Figure 4. ORTEP views of 1 at $300 \mathrm{~K}$. The grey, green and yellow represents the carbon, chlorine and sulfur atoms, respectively. Hydrogen atoms have been omitted for clarity.

Molecules of dyads $\mathbf{1}$ and $\mathbf{2}$ are arranged in a head-to-tail manner, as shown in Figures 5 and 6, with short $\mathrm{Cl} \cdots \mathrm{Cl}$ and S... Cl contacts of 3.3 and $3.4 \AA$, respectively. Additionally, there are short $\mathrm{C} \cdots \mathrm{C}$ interactions of $2.4 \AA$ between adjacent phenyl units of the bridge. It is important to mention that the TTF moieties are not disposed in a herringbone manner with short S...S interactions as it was observed for MPTTF-PTM radical dyad. ${ }^{[20]}$ 


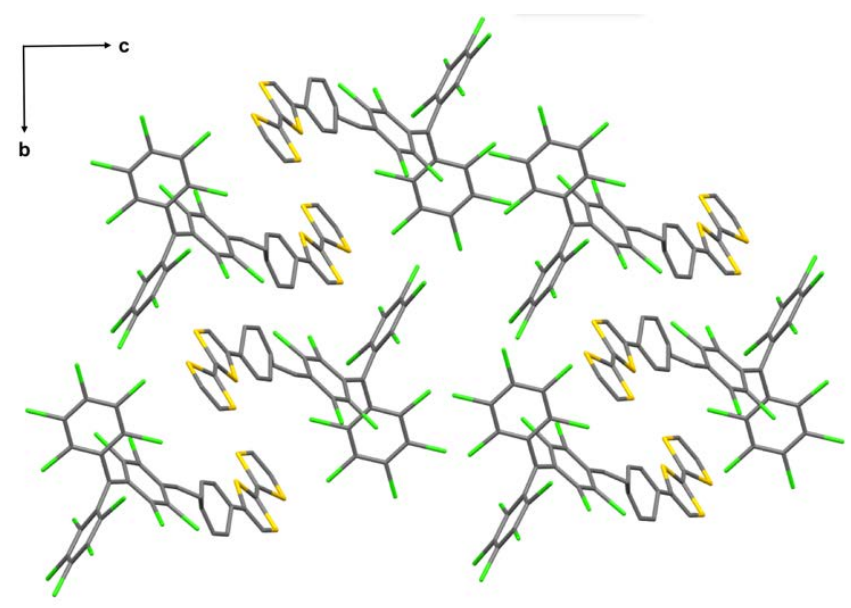

Figure 5. Molecular packing of $\mathbf{1}$ (and the isomorphous 2) in the $b c$ plane.

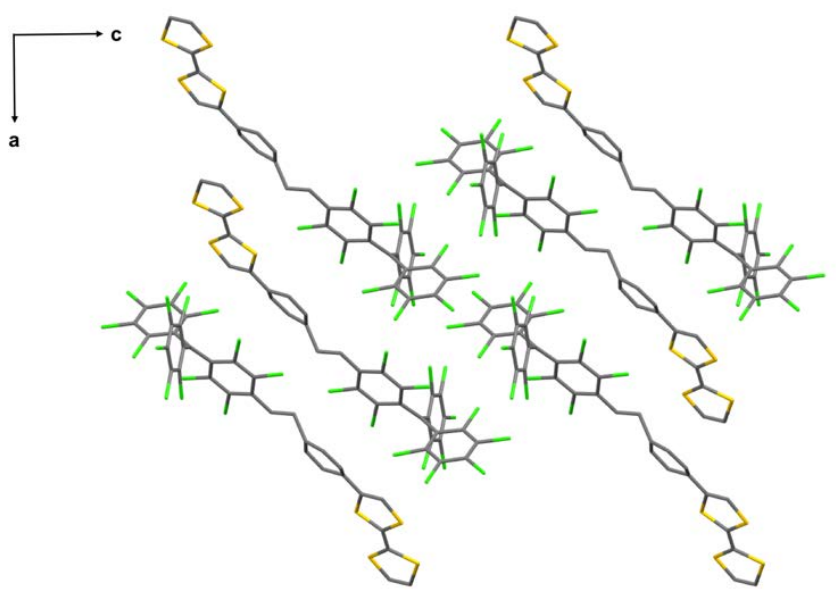

Figure 6. Molecular packing of $\mathbf{1}$ (and the isomorphous 2 ) in the ac plane.

Single-crystal synchrotron-radiation diffraction experiments for dyad 1 at 4 different pressures up to $11.5 \mathrm{GPa}$ were performed at the MS beamline of the Swiss Light Source. ${ }^{[29]}$ Data at 2.0 GPa could also be used for a structural refinement, while a continuous decay in the signal strength, possibly due to radiation damage, did not allow for full refinement at higher pressure values. The relative compression data, shown in Figure 7, shows no major discontinuity and is consistent with the initial cell. Reconstructed precession images at 2 and $11.5 \mathrm{GPa}$ (see Figure S6) also do not present any marked difference, at the side of the volume contraction, with respect to each other, showing no sign of a different metric. The main difference seems to be the quenching of the disordered position in the phenyl group, which becomes disordered in two positions in its own plane, rather than off plane, as evident in the refined structure at $2.0 \mathrm{GPa}$.

Resistivity measurements on crystals of $\mathbf{1}$ and $\mathbf{2}$ were performed under high-pressure conditions. Pressure and temperature dependence measurements of the resistivity of radical dyad 1 were performed with three independent crystals showing an insulating behavior at ambient pressure. In MPTTFPTM dyad, ${ }^{[20,30]}$ increasing the pressure up to $11.5 \mathrm{GPa}$ the room-temperature resistivity of such crystals rapidly decreased exhibiting a semiconducting behavior with an activation energy $\left(E_{\mathrm{a}}\right)$ of $0.2 \mathrm{eV}$ (Figure 8). On the contrary, three independent crystals of non-radical dyad 2 were measured up to $21 \mathrm{GPa}$ and they were found always to be insulating under these conditions. The room-temperature conductivity $\left(\sigma_{\mathrm{rt}}\right)$ of radical dyad 1 continuously increased with a reversible behavior exhibiting a value of $9 \cdot 10^{-3} \mathrm{~S} \mathrm{~cm}^{-1}$ at $14.6 \mathrm{GPa}$ with a $E_{\mathrm{a}}$ of $0.068 \mathrm{eV}$. Interestingly, to compare with the results that were obtained from high pressure measurements on MPTTF-PTM (at 15.2 GPa, $\sigma_{\mathrm{rt}}$ and $E_{\mathrm{a}}$ were $0.76 \mathrm{~S} \mathrm{~cm}^{-1}$ and $0.067 \mathrm{eV}$, respectively), ${ }^{[20]}$ radical dyad 1 showed almost the same value of $E_{\mathrm{a}}$, but $\sigma_{\mathrm{rt}}$ was almost two orders of magnitude smaller. As discussed for MPTTF-PTM crystals, due to the incorporation of TTF units where the MPTTF units are stacked along $b$ axis, the conductivity increases with the enhancement of the intermolecular overlap between the MPTTF-PTM molecules. However, in radical dyad $\mathbf{1}$ the packing of the TTF units does not have the same impact on the intermolecular overlap giving rise to a smaller conductivity than in the MPTTF-PTM. In addition, a pressure induced semiconducting state appears at higher pressure. Reversibility and reproducibility of the experiments are shown in the Supporting Information (Figures S7, S8, and S9).

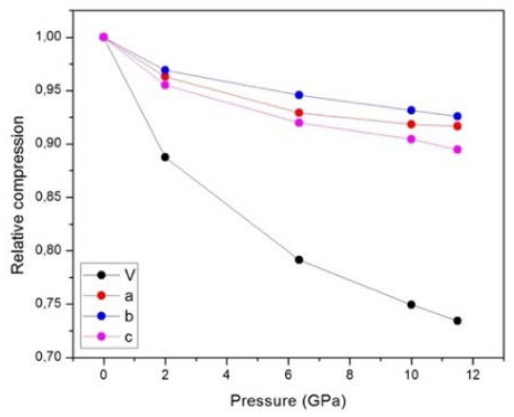

Figure 7. Evolution of experimental crystal cell parameters of radical dyad $\mathbf{1}$ at different pressures.

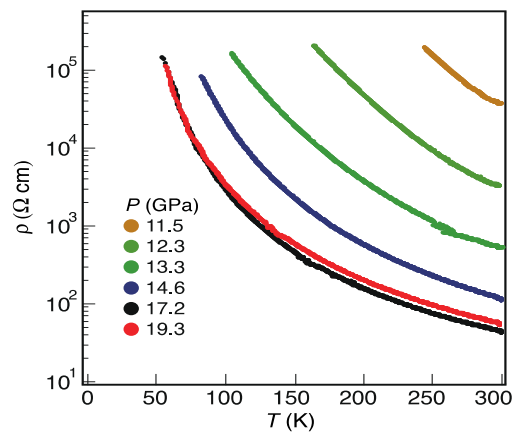

Figure 8. Temperature dependence of the resistivity of radical dyad $\mathbf{1}$ at different pressures. 
We used density functional theory to investigate the electronic structure of the two isostructural materials TTF-Ph-PTM radical dyad 1 and the non-radical dyad 2. The phenylene vinylene bridge $(\mathrm{Ph})$ is slightly disordered; two configurations of almost equal probability are tilted by about 30 degree against each other. In the radicals, there is also a slight disorder in the TTF part of the molecule so we perform the calculations for the majority configuration. We relaxed the hydrogen positions, most of which are calculated rather than measured in the experimental structure. We calculate the electronic structure with the all electron full potential local orbital (FPLO) basis ${ }^{[31]}$ with the generalized gradient approximation $(\mathrm{GGA})^{[32]}$ exchange and correlation functional (see Figure 9).

(a) TTF-Ph-PTM-H

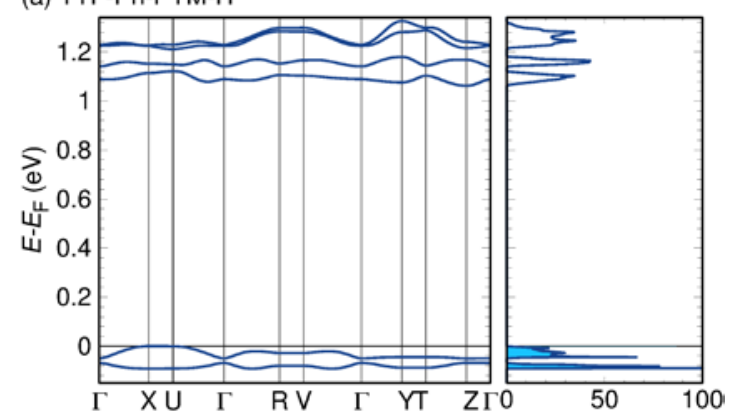

(b) TTF-Ph-PTM radical (spin polarized) DOS (states/eV/f.u.)

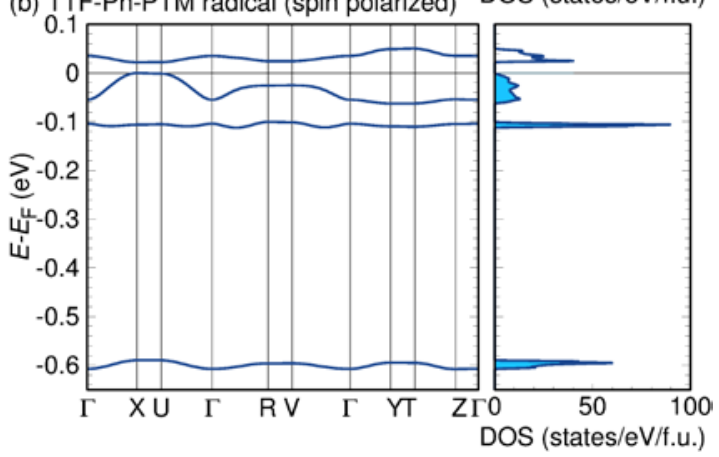

Figure 9. Electronic bands and density of states of (a) TTF-Ph-PTM-H (2) and (b) TTF-Ph-PTM radical (1) calculated using density functional theory. Occupied states are marked in the DOS. TTF-Ph-PTM-H is a band insulator with a large gap of $E_{g}=1.06 \mathrm{eV}$. For the TTF-Ph-PTM radical, spin-polarized calculations are used, and the electronic structure corresponds to a magnetic solution with zero total magnetic moment.

In the case of TTF-Ph-PTM-H (2), we find a band insulator with a gap of $E_{\mathrm{g}}=1.06 \mathrm{eV}$. However, at the non-spin-polarized GGA level, TTF-Ph-PTM radical dyad (1) has two extra bands at the Fermi level which are half-filled (see Figure S10). We can use band weights to determine that the central $C$ atoms of the two PTM radicals in the unit cell $(Z=2)$ provide the main contribution to the half-filled bands. It is important to note that the TTF-PhPTM radical dyad $\mathbf{1}$ is a Mott insulator at ambient pressure (it is half-filled band and the ratio between electronic bandwidth $W$ and Coulomb repulsion is of the order of 1 ) with a similar behaviour than the previously reported MPTTF-PTM radical dyad. ${ }^{[20]}$ As the open shells of the radicals likely give rise to magnetism, we also performed spin-polarized calculations for the TTF-Ph-PTM radical $\mathbf{1}$. The simplest way to do this is by retaining the $P 1$ space group which makes the two radicals in the unit cell symmetry equivalent and allows only for a ferromagnetic solution. This solution is $82 \mathrm{meV}$ per radical lower in energy than the nonmagnetic solution. Each of the molecules carries exactly $S=1 / 2$. The electronic structure is shown in the SI (Figure S11). The more realistic case of an antiferromagnetic solution with zero total magnetization requires breaking inversion symmetry; the corresponding calculations converge with a moment of $0.51 \mu_{\mathrm{B}}$ per radical. The total energy is 0.5 meV per radical below the ferromagnetic solution, indicating that a state with zero net magnetic moment is the ground state, and that the effective exchange interaction between radicals is antiferromagnetic. The electronic structure of the antiferromagnetic solution is shown in Figure 9 (b). The antiferromagnetic solution is insulating with a small gap of $E_{\mathrm{g}}=$ $22 \mathrm{meV}$. While we cannot describe, using simple DFT calculations, the magnetically disordered state that is most likely realized for temperatures exceeding a few Kelvin, we can clearly establish that the TTF-Ph-PTM radical 1 has a semiconducting ground state with a very small activation energy.

In summary, we have reported the synthesis and electronic properties of a new organic non-planar donor-acceptor dyad based on the electron-donor TTF linked to the electron-acceptor PTM moiety in its radical (1) and non-radical (2) forms. Interestingly, the radical dyad $\mathbf{1}$ exhibited semiconducting behaviour under high pressure whereas the isostructural nonradical analogue 2 was insulating at all measured pressure. Spin-polarized DFT band calculations confirmed that non-radical dyad 2 is a band insulator with a large gap of $1 \mathrm{eV}$ whereas radical dyad 1 has a semiconducting ground state with a small gap. This study shows that tuning the electronic structure (reducing the HOMO-SUMO gap) of these family of radical dyads by decreasing the bridge length between the acceptor and donor units is a good strategy to enhance the transport properties and has to be taken into account in the rational design of neutral radical conductors.

\section{Acknowledgements}

This work was supported the Spanish MINECO/FEDER grants ("Mother"; MAT 2016-80826-R and "Fancy"; CTQ 2016-80030$\mathrm{R})$, the Networking Research Center on Bioengineering, Biomaterials, and Nanomedicine (CIBER-BBN), and the AGAUR, Generalitat de Catalunya (grant 2014-SGR-17), by the EU Marie Curie ITN iSwitch (grant 642196) and by Swiss National Science Foundation, project 200020_162861. ICMAB is supported by the Spanish Ministry of Economy and Competitiveness, through the "Severo Ochoa" Programme for Centres of Excellence in R\&D (SEV- 2015-0496). M.S. is grateful to Spanish Ministerio de Educación, Cultura y Deporte for a FPU. HO Jeschke, and R. Valenti thank the Deutsche Forschungsgemeinschaft (DFG) for funding through grant SFB/TRR49 and Steve Winter for useful discussions. We thank Xavier Fontrodona (Univ. Girona) for single-crystal $\mathrm{X}$-ray diffraction measurements. We also thank Amable Bernabé and Vega Lloveras (ICMAB-CSIC) for MALDI and ESR spectroscopy. 
Keywords: Single-component conductor • Organic radical • Donor-acceptor • Tetrathiafulvalene $\bullet$ Polychlorotriphenylmethyl
[31] K. Koepernik, H. Eschrig, Phys. Rev. B 1999, 59, 1743.

[32] J. P. Perdew, K. Burke, M. Ernzerhof, Phys. Rev. Lett. 1996, 77 , 3865-3868.

[1] P. Batail, Chem. Rev. 2004, 104, 4887-4890.

[2] A. Kobayashi, E. Fujiwara, H. Kobayashi, Chem. Rev. 2004, 104, 5243-5264.

[3] H. Tanaka, Y. Okano, H. Kobayashi, Science 2001, 291, 285-288.

[4] D. Belo, H. Alves, E. B. Lopes, M. T. Duarte, V. Gama, R. T. Henriques, M. Almeida, A. Pérez-Benítez, C. Rovira, J. Veciana Chem. Eur. J. 2001, 7, 511-519.

[5] R. Llusar, S. Uriel, C. Vicent, J. M. Clemente-Juan, E. Coronado, C. J. Gómez-García, B. Braïda, E. Canadell, J. Am. Chem. Soc. 2004 126, 12076-12083.

[6] H. Cui, H. Kobayashi, S. Ishibashi, M. Sasa, F. Iwase, R. Kato, A. Kobayashi, J. Am. Chem. Soc. 2014, 136, 7619-7622.

[7] D. G. Branzea, F. Pop, P. Auban-Senzier, R. Clérac, P. Alemany, E. Canadell, N. Avarvari, J. Am. Chem. Soc. 2016, 138, 6838-6851.

[8] T. Isono, H. Kamo, A. Ueda, K. Takahashi, A. Nakao, R. Kumai, H. Nakao, K. Kobayashi, Y. Murakami, H. Mori, Nat. Commun. 2013, 4 1344-1346.

[9] A. Ueda, S. Yamada, T. Isono, H. Kamo, A. Nakao, R. Kumai, H. Nakao, Y. Murakami, K. Yamamoto, Y. Nishio, et al., J. Am. Chem. Soc. 2014, 136, 12184-12192.

[10] Y. Kobayashi, T. Terauchi, S. Sumi, Y. Matsushita, Nat. Mater. 2017 16, 109-115.

[11] R. C. Haddon, Nature 1975, 256, 394-396.

[12] S. K. Pal, M. E. Itkis, F. S. Tham, R. W. Reed, R. T. Oakley, R. C. Haddon, J. Am. Chem. Soc. 2008, 130, 3942-3951.

[13] S. K. Pal, P. Bag, A. Sarkar, X. Chi, M. E. Itkis, F. S. Tham, B. Donnadieu, R. C. Haddon, J. Am. Chem. Soc. 2010, 132, 1725817264.

[14] S. K. Pal, P. Bag, M. E. Itkis, F. S. Tham, R. C. Haddon, J. Am. Chem. Soc. 2014, 136, 14738-14741.

[15] X. Yu, A. Mailman, K. Lekin, A. Assoud, C. M. Robertson, B. C. Noll, C. F. Campana, J. a K. Howard, P. a. Dube, R. T. Oakley, J. Am. Chem. Soc. 2012, 134, 2264-2275.

[16] A. Mailman, S. M. Winter, X. Yu, C. M. Robertson, W. Yong, J. S. Tse, R. a. Secco, Z. Liu, P. a. Dube, J. a K. Howard, et al., J. Am. Chem. Soc. 2012, 134, 9886-9889.

[17] J. W. L. Wong, A. Mailman, K. Lekin, S. M. Winter, W. Yong, J. Zhao, S. V. Garimella, J. S. Tse, R. a. Secco, S. Desgreniers, et al., J. Am. Chem. Soc. 2014, 136, 1070-1081.

[18] D. Tian, S. M. Winter, A. Mailman, J. W. L. Wong, W. Yong, H. Yamaguchi, Y. Jia, J. S. Tse, S. Desgreniers, R. A. Secco, et al., J. Am. Chem. Soc. 2015, 137, 14136-14148.

[19] A. Mailman, A. A. Leitch, W. Yong, E. Steven, S. M. Winter, R. C. M. Claridge, A. Assoud, J. S. Tse, S. Desgreniers, R. A. Secco, et al., J. Am. Chem. Soc. 2017, 139, 2180-2183.

[20] M. Souto, H. Cui, M. Peña-Álvarez, V. G. Baonza, H. O. Jeschke, M. Tomic, R. Valent, D. Blasi, I. Ratera, C. Rovira, et al., J. Am. Chem. Soc. 2016, 138, 11517-11525.

[21] J. Guasch, L. Grisanti, V. Lloveras, J. Vidal-Gancedo, M. Souto, D. C. Morales, M. Vilaseca, C. Sissa, A. Painelli, I. Ratera, et al., Angew. Chem. Int. Ed. 2012, 51, 11024-11028.

[22] J. Guasch, L. Grisanti, M. Souto, V. Lloveras, J. Vidal-Gancedo, I. Ratera, A. Painelli, C. Rovira, J. Veciana, J. Am. Chem. Soc. 2013 135, 6958-6967

[23] M. Souto, M. V. Solano, M. Jensen, D. Bendixen, F. Delchiaro, A. Girlando, A. Painelli, J. O. Jeppesen, C. Rovira, I. Ratera, et al. Chem. Eur. J. 2015, 21, 8816-8825.

[24] M. Souto, J. Calbo, I. Ratera, E. Ortí, J. Veciana, Chem. Eur. J. 2017, 23, $11067-11075$.

[25] S. Vela, M. Souto, I. Ratera, C. Rovira, J. Veciana, J. Phys. Chem A 2016, 120, 10297-10303.

[26] Y. Mitamura, H. Yorimitsu, K. Oshima, A. Osuka, Chem. Sci. 2011 2, 2017.

[27] C. Rovira, D. Ruiz-Molina, O. Elsner, J. Vidal-Gancedo, J. Bonvoisin J. P. Launay, J. Veciana, Chem. Eur. J. 2001, 637-639, 251-257. M. Souto, D. Bendixen, M. Jensen, V. Díez-Cabanes, J. Cornil, J. Jeppesen, I. Ratera, C. Rovira, J. Veciana, Magnetochemistry 2016 $2,46$.

[29] P. R. Willmott, D. Meister, S. J. Leake, M. Lange, A. Bergamaschi, M. Böge, M. Calvi, C. Cancellieri, N. Casati, A. Cervellino, et al., J. Synchrotron Radiat. 2013, 20, 667-682.

[30] M. Souto, C. Rovira, I. Ratera, J. Veciana, CrystEngComm 2017, 19 197-206. 


\section{COMMUNICATION}

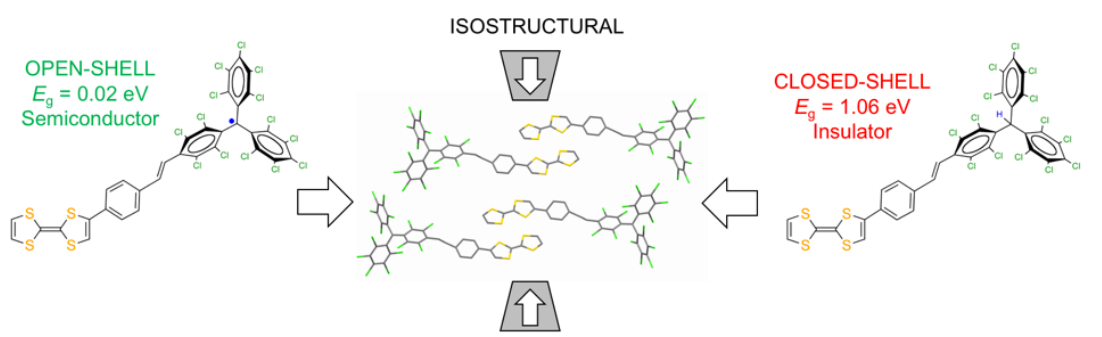

We present here a nonplanar and spin localised radical (1) based on a tetrathiafulvalene (TTF) moiety, linked to a perchlorotriphenylmethyl (PTM) radical by a conjugated bridge, which exhibits a semiconducting behaviour upon application of high pressure. On the contrary, the isostructural non-radical derivative 2 shows an insulating behaviour at all measured pressures. The different electronic structures of these two isostructural systems have a direct influence on the conducting properties as demonstrated by band structure DFT calculations.
Manuel Souto, Maria Chiara Gullo, HengBo Cui, Nicola Casati, Fabio Montisci, Harald O. Jeschke, Roser Valentí, Imma Ratera, Concepció Rovira and Jaume Veciana*

Page No. - Page No.

Role of the open-shell character in the pressure-induced conductivity in an organic donor-acceptor dyad 